\title{
CO-T-STRUCTURES: THE FIRST DECADE
}

\author{
PETER JØRGENSEN
}

\begin{abstract}
Co-t-structures were introduced about ten years ago as a type of mirror image of t-structures. Like t-structures, they permit to divide an object in a triangulated category $\mathscr{T}$ into a "left part" and a "right part", but there are crucial differences. For instance, a bounded t-structure gives rise to an abelian subcategory of $\mathscr{T}$, while a bounded co-tstructure gives rise to a so-called silting subcategory.

This brief survey will emphasise three philosophical points. First, bounded t-structures are akin to the canonical example of "soft" truncation of complexes in the derived category. Secondly, bounded co-t-structures are akin to the canonical example of "hard" truncation of complexes in the homotopy category.

Thirdly, a triangulated category $\mathscr{T}$ may be skewed towards t-structures or co-t-structures, in the sense that one type of structure is more useful than the other for studying $\mathscr{T}$. In particular, we think of derived categories as skewed towards t-structures, and of homotopy categories as skewed towards co-t-structures.
\end{abstract}

\section{INTRODUCTION}

The notion of co-t-structure in a triangulated category $\mathscr{T}$ was introduced independently by Bondarko and Pauksztello, see Definition 2.1. It is a mirror image of the classic notion of t-structure due to Beilinson, Bernstein, and Deligne, see Definition 1.1.

Given an object $t \in \mathscr{T}$, both types of structure give a way to divide $t$ into a "left part" and a "right part". This is exemplified by dividing a complex of modules into a left and a right part by "soft" or "hard" truncation, see Figures 11 and 2 .

Each case gives a triangle $u \rightarrow t \rightarrow v$. Crucially, for a t-structure, $u$ is the left part of $t$ and $v$ the right part; for a co-t-structure, vice versa. This reversal leads to a number of differences, and the theories of t-structures and co-t-structures are far from being simple mirrors of each other. For instance, while a bounded t-structure induces an abelian subcategory of $\mathscr{T}$, a bounded co-t-structure induces a so-called silting subcategory; see Definition 2.3.

In the decade since their inception, the theory of co-t-structures has grown considerably. This brief survey is far from encyclopedic, but has the important goal of communicating three philosophical points:

(i) Bounded t-structures are akin to soft truncation in the bounded derived category of an abelian category.

(ii) Bounded co-t-structures are akin to hard truncation in the bounded homotopy category of an additive category.

2010 Mathematics Subject Classification. 18E30.

Key words and phrases. Abelian subcategory, Auslander-Reiten quiver, co-heart, co-t-structure, complex of modules, derived category, heart, homotopy category, silting mutation, silting quiver, silting subcategory, simple minded collection, t-structure, triangulated category, truncation. 


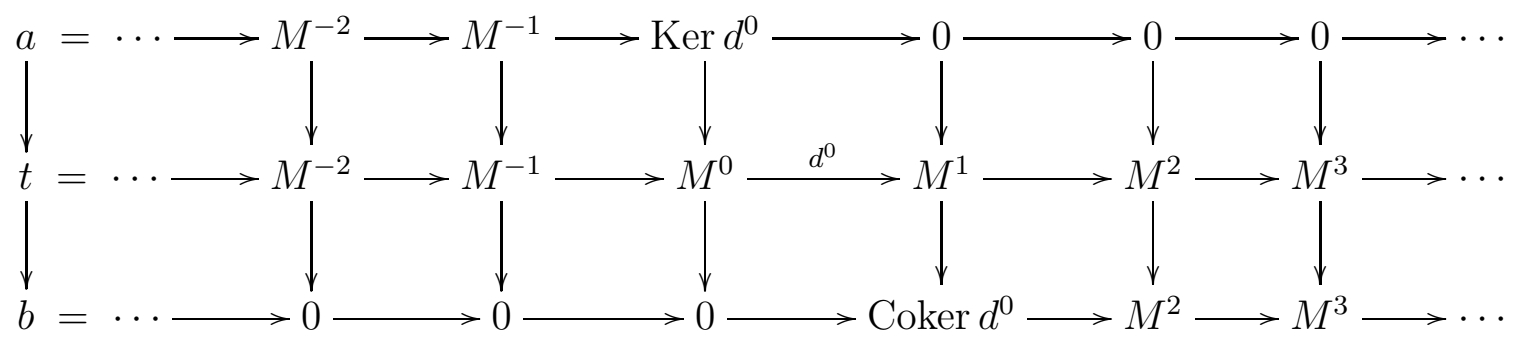

Figure 1. A complex $t=\cdots \rightarrow M^{-1} \rightarrow M^{0} \stackrel{d^{0}}{\rightarrow} M^{1} \rightarrow M^{2} \rightarrow \cdots$ has soft truncations $a$ and $b$. There is a triangle $a \rightarrow t \rightarrow b$ in the derived category. Each vertical module homomorphism is either the identity, a canonical inclusion or surjection, or zero.

(iii) A triangulated category $\mathscr{T}$ may be skewed in the direction of t-structures or co-tstructures, in the sense that one type of structure is more useful than the other for studying $\mathscr{T}$.

We explain these points in the next three subsections. Throughout, $\mathscr{T}$ is a triangulated category with Hom-spaces $\mathscr{T}(-,-)$ and suspension functor $\Sigma$. If $\mathscr{M}$ is an abelian category then $\mathscr{D}^{\mathrm{b}}(\mathscr{M})$ is the derived category of bounded complexes over $\mathscr{M}$, and if $\mathscr{P}$ is an additive category then $\mathscr{K}^{\mathrm{b}}(\mathscr{P})$ is the homotopy category of bounded complexes over $\mathscr{P}$.

(i) Bounded t-structures are akin to soft truncation in the bounded derived category. Let $R$ be a ring, $\mathscr{M}=\operatorname{Mod} R$ the category of left modules over $R$. Each complex $t \in \mathscr{D}^{\mathrm{b}}(\mathscr{M})$ has soft truncations $a$ and $b$ as shown in Figure 1, and there is a triangle $a \rightarrow t \rightarrow b$ in $\mathscr{D}^{\mathrm{b}}(\mathscr{M})$. The full subcategories $\mathscr{A}$ and $\mathscr{B}$ consisting of complexes isomorphic to such truncations satisfy Definition 1.1 and hence form a bounded t-structure, sometimes known as the standard t-structure, see Example 1.2.

Up to isomorphism, the heart $\mathscr{H}=\mathscr{A} \cap \sum \mathscr{B}$ consists of complexes concentrated in degree 0 . The heart is an abelian subcategory of $\mathscr{D}^{\mathrm{b}}(\mathscr{M})$ which is equivalent to $\mathscr{M}$. Each $t \in \mathscr{D}^{\mathrm{b}}(\mathscr{M})$ permits a "tower" as shown in Proposition 3.1 due to Beilinson, Bernstein, and Deligne. This expresses how to build $t$ from objects of the form $\Sigma^{i} h$ with $h \in \mathscr{H}$. The objects can be taken to be $\Sigma^{i} \mathrm{H}^{-i}(t)$ and then the tower shows how $t$ is built from its cohomology modules, see Example 3.3.

In general, if $(\mathscr{A}, \mathscr{B})$ is a bounded t-structure in a triangulated category $\mathscr{T}$, then each $t \in \mathscr{T}$ still permits a triangle $a \rightarrow t \rightarrow b$ with $a \in \mathscr{A}, b \in \mathscr{B}$. The heart $\mathscr{H}$ is still an abelian subcategory of $\mathscr{T}$ by Theorem 1.3 due to Beilinson, Bernstein, and Deligne, and each $t \in \mathscr{T}$ still has the tower in Proposition 3.1 .

Working in such a setup is akin to working with soft truncation in $\mathscr{D}^{\mathrm{b}}(\mathscr{M})$.

(ii) Bounded co-t-structures are akin to hard truncation in the bounded homotopy category. Let $R$ be a ring, $\mathscr{P}=\operatorname{Prj} R$ the category of projective left modules over $R$. Each complex $t \in \mathscr{K}^{\mathrm{b}}(\mathscr{P})$ has hard truncations $x$ and $y$ as shown in Figure 2, and there is a triangle $x \rightarrow t \rightarrow y$ in $\mathscr{K}^{\mathrm{b}}(\mathscr{P})$. The full subcategories $\mathscr{X}$ and $\mathscr{Y}$ consisting of 


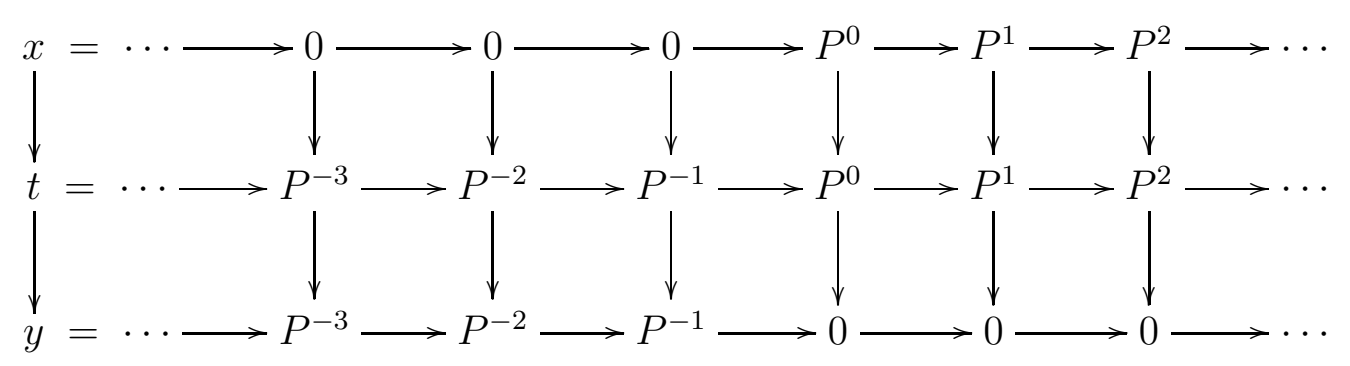

Figure 2. A complex $t=\cdots \rightarrow P^{-2} \rightarrow P^{-1} \rightarrow P^{0} \rightarrow P^{1} \rightarrow \cdots$ has hard truncations $x$ and $y$. There is a triangle $x \rightarrow t \rightarrow y$ in the homotopy category. Each vertical module homomorphism is the identity or zero.

complexes isomorphic to such truncations satisfy Definition 2.1 and hence form a bounded co-t-structure, sometimes known as the standard co-t-structure, see Example 2.2.

Up to isomorphism, the co-heart $\mathscr{C}=\mathscr{X} \cap \Sigma^{-1} \mathscr{Y}$ consists of complexes concentrated in degree 0 . The co-heart is an additive subcategory of $\mathscr{K}^{\mathrm{b}}(\mathscr{P})$ which is equivalent to $\mathscr{P}$.

The co-heart is not in general abelian, but it does have the strong property of being a socalled silting subcategory of $\mathscr{K}^{\mathrm{b}}(\mathscr{P})$, see Definition 2.3 and Theorem 2.4 due to Mendoza Hernández et.al. Such subcategories are structurally important.

Each $t=\cdots \rightarrow P^{-3} \rightarrow P^{-2} \rightarrow P^{-1} \rightarrow P^{0} \rightarrow P^{1} \rightarrow P^{2} \rightarrow \cdots$ in $\mathscr{K}^{\mathrm{b}}(\mathscr{P})$ permits a "tower" as shown in Proposition 3.2 due to Bondarko. This expresses how to build $t$ from objects of the form $\Sigma^{i} c$ with $c \in \mathscr{C}$. The objects can be taken to be $\Sigma^{i} P^{-i}$ and then the tower shows how $t$ is built from its constituent modules, see Example 3.4 .

In general, if $(\mathscr{X}, \mathscr{Y})$ is a bounded co-t-structure in a triangulated category $\mathscr{T}$, then each $t \in \mathscr{T}$ still permits a triangle $x \rightarrow t \rightarrow y$ with $x \in \mathscr{X}, y \in \mathscr{Y}$. The co-heart $\mathscr{C}$ is still a silting subcategory of $\mathscr{T}$ by Theorem 2.4, and each $t \in \mathscr{T}$ still has the tower in Proposition 3.2 .

Working in such a setup is akin to working with hard truncation in $\mathscr{K}^{\mathrm{b}}(\mathscr{P})$.

(iii) Triangulated categories skewed in the direction of t-structures or co-tstructures. When studying a given triangulated category, bounded co-t-structures may be more useful than bounded t-structures, simply because there are none of the latter. See Section 4 for a toy example.

Section 5 shows a subtler skewing phenomenon. Let $\Lambda$ be a finite dimensional $\mathbb{C}$-algebra, $\mathscr{M}=\bmod \Lambda$ the category of finite dimensional left modules over $\Lambda$, and $\mathscr{P}=\operatorname{prj} \Lambda$ the category of finite dimensional projective left modules over $\Lambda$. Theorem 5.2, due to König and Yang, shows a bijection between all bounded co-t-structures in $\mathscr{K}^{\mathrm{b}}(\mathscr{P})$ and the bounded tstructures in $\mathscr{D}^{\mathrm{b}}(\mathscr{M})$ whose hearts are length categories (these are, in a sense, the "algebraic" t-structures; for instance, they permit a nice mutation theory).

In itself, this does not imply that either category has more t-structures than co-t-structures or vice versa, but we think of it as indicating that when going from derived to homotopy categories, the role played by t-structures is taken over by co-t-structures.

Summing up, Sections 1 and 2 show the definitions of t-structures and co-t-structures, emphasising the similarities with soft and hard truncation of complexes of modules. Section 
3 shows the "towers" whereby general objects can be built from objects in the (co-)heart. Sections 4 and 5 illustrate how a triangulated category may be skewed towards t-structures or co-t-structures.

Section 6 shows the silting mutation of Aihara and Iyama which mutates between bounded co-t-structures. It permits the definition of the so-called silting quiver which is a combinatorial picture of how the bounded co-t-structures fit inside a fixed triangulated category.

\section{T-STRUCTURES}

The following definition was made in [4, def. 1.3.1].

Definition 1.1 (Beilinson, Bernstein, and Deligne). A $t$-structure in the triangulated category $\mathscr{T}$ is a pair $(\mathscr{A}, \mathscr{B})$ of full subcategories, closed under isomorphisms, direct sums, and direct summands, which satisfy the following conditions.

(i) $\Sigma \mathscr{A} \subseteq \mathscr{A}$ and $\Sigma^{-1} \mathscr{B} \subseteq \mathscr{B}$.

(ii) $\mathscr{T}(\mathscr{A}, \mathscr{B})=0$.

(iii) For each object $t \in \mathscr{T}$ there is a triangle $a \rightarrow t \rightarrow b$ with $a \in \mathscr{A}, b \in \mathscr{B}$.

The heart is $\mathscr{H}=\mathscr{A} \cap \sum \mathscr{B}$.

The t-structure is called bounded if

$$
\bigcup_{i \in \mathbb{Z}} \Sigma^{i} \mathscr{A}=\bigcup_{i \in \mathbb{Z}} \Sigma^{i} \mathscr{B}=\mathscr{T}
$$

The objects $a$ and $b$ in Definition 1.1(iii) depend functorially on $t$. The resulting functor $t \mapsto a$ is a right-adjoint to the inclusion $\mathscr{A} \hookrightarrow \mathscr{T}$. Similarly, $t \mapsto b$ is a left adjoint to the inclusion $\mathscr{B} \hookrightarrow \mathscr{T}$. See [4, prop. 1.3.3].

The following is the canonical example of a t-structure.

Example 1.2 (The standard t-structure). Let $R$ be a ring, $\mathscr{M}=\operatorname{Mod} R$ the category of left modules over $R$. Let $\mathscr{A}$ and $\mathscr{B}$ be the isomorphism closures in the bounded derived category $\mathscr{D}^{\mathrm{b}}(\mathscr{M})$ of the subsets

$$
\begin{aligned}
& \left\{\cdots \rightarrow M^{-2} \rightarrow M^{-1} \rightarrow M^{0} \rightarrow 0 \longrightarrow 0 \longrightarrow 0 \rightarrow \cdots \mid M^{i} \in \mathscr{M}, M^{i}=0 \text { for } i \ll 0\right\}, \\
& \left\{\cdots \rightarrow 0 \longrightarrow 0 \longrightarrow 0 \rightarrow M^{1} \rightarrow M^{2} \rightarrow M^{3} \rightarrow \cdots \mid M^{i} \in \mathscr{M}, M^{i}=0 \text { for } i \gg 0\right\} .
\end{aligned}
$$

We will show that if $\mathscr{A}$ and $\mathscr{B}$ are viewed as full subcategories, then $(\mathscr{A}, \mathscr{B})$ is a bounded t-structure with heart $\mathscr{H}$ equivalent to $\mathscr{M}$.

It is easy to show

$$
\begin{aligned}
& \mathscr{A}=\left\{M \in \mathscr{D}^{\mathrm{b}}(\mathscr{M}) \mid \mathrm{H}^{i}(M)=0 \text { for } i \geqslant 1\right\}, \\
& \mathscr{B}=\left\{M \in \mathscr{D}^{\mathrm{b}}(\mathscr{M}) \mid \mathrm{H}^{i}(M)=0 \text { for } i \leqslant 0\right\} .
\end{aligned}
$$

This description clearly implies that $\mathscr{A}$ and $\mathscr{B}$ are closed under isomorphisms, direct sums, and direct summands. 
We next check the conditions in Definition 1.1. Condition (i) is immediate. Condition (ii) requires that if objects

$$
\begin{aligned}
& a=\cdots \rightarrow M^{-2} \rightarrow M^{-1} \rightarrow M^{0} \rightarrow 0 \longrightarrow 0 \longrightarrow 0 \rightarrow \cdots, \\
& b=\cdots \longrightarrow 0 \longrightarrow 0 \longrightarrow 0 \rightarrow M^{1} \rightarrow M^{2} \rightarrow M^{3} \rightarrow \cdots
\end{aligned}
$$

in $\mathscr{A}$ and $\mathscr{B}$ are given, then $\operatorname{Hom}_{\mathscr{D} \mathrm{b}(\mathscr{M})}(a, b)=0$. This can be shown by noting that $a$ has a projective resolution

$$
p=\cdots \rightarrow P^{-2} \rightarrow P^{-1} \rightarrow P^{0} \rightarrow 0 \rightarrow 0 \rightarrow 0 \rightarrow \cdots,
$$

and that

$$
\operatorname{Hom}_{\mathscr{D}}(\mathscr{M})(a, b)=\operatorname{Hom}_{\mathscr{K}(\mathscr{M})}(p, b)=0 .
$$

Here $\mathscr{K}(\mathscr{M})$ is the homotopy category of complexes over $\mathscr{M}$, and the second $=$ holds because in each degree, either the complex $p$ or the complex $b$ is zero. The triangle in Definition1.1(iii) can be obtained by soft truncation of the object $t=\cdots \rightarrow M^{-1} \rightarrow M^{0} \stackrel{d^{0}}{\rightarrow} M^{1} \rightarrow M^{2} \rightarrow \cdots$ in $\mathscr{D}^{\mathrm{b}}(\mathscr{M})$, see Figure 1 in the introduction.

It is immediate that the t-structure $(\mathscr{A}, \mathscr{B})$ is bounded.

Finally, it follows from Equation (1.1) that the heart $\mathscr{H}=\mathscr{A} \cap \sum \mathscr{B}$ is

$$
\mathscr{H}=\left\{M \in \mathscr{D}^{\mathrm{b}}(\mathscr{M}) \mid \mathrm{H}^{i}(M)=0 \text { for } i \neq 0\right\},
$$

and this subcategory of $\mathscr{D}^{\mathrm{b}}(\mathscr{M})$ is equivalent to $\mathscr{M}$.

The following pivotal result is one of the motivations for the definition of t-structures. It was proved in [4, thm. 1.3.6].

Theorem 1.3 (Beilinson, Bernstein, and Deligne). Let $(\mathscr{A}, \mathscr{B})$ be a t-structure in $\mathscr{T}$. Then the heart $\mathscr{H}=\mathscr{A} \cap \Sigma \mathscr{B}$ is an abelian subcategory of $\mathscr{T}$.

Example 1.4. Let $\Lambda=\mathbb{C} A_{2}$ be the path algebra of the quiver

$$
A_{2}=1 \rightarrow 2
$$

and let $\mathscr{M}=\bmod \Lambda$ be the category of finite dimensional left modules over $\Lambda$.

There is a bounded t-structure $(\mathscr{A}, \mathscr{B})$ in $\mathscr{D}^{\mathrm{b}}(\mathscr{M})$ defined by Equation (1.1); this is shown by the same method as in Example 1.2.

There are three isomorphism classes of indecomposable objects in $\mathscr{M}$ given by the following representations of the quiver $A_{2}$.

$$
0 \rightarrow \mathbb{C}, \quad \mathbb{C} \stackrel{\text { id }}{\rightarrow} \mathbb{C}, \quad \mathbb{C} \rightarrow 0
$$

They induce isomorphism classes $x_{0}, x_{1}, x_{2}$ of indecomposable objects in $\mathscr{D}^{\mathrm{b}}(\mathscr{M})$, and we define further isomorphism classes recursively by $\Sigma x_{i}=x_{i+3}$ for $i \in \mathbb{Z}$.

The Auslander-Reiten quiver of $\mathscr{D}^{\mathrm{b}}(\mathscr{M})$ looks as follows, where red and green vertices show $\mathscr{A}$ and $\mathscr{B}$.

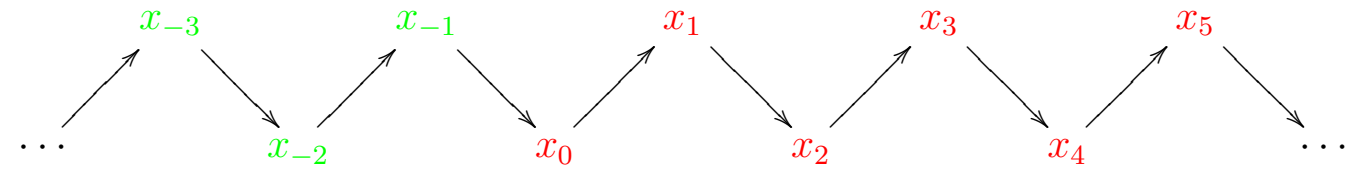

Note that if $t \in \mathscr{D}^{\mathrm{b}}(\mathscr{M})$ is indecomposable, then $t$ is in $\mathscr{A}$ or in $\mathscr{B}$, so the triangle in Definition 1.1(iii) is trivial in the sense that it reads $t \rightarrow t \rightarrow 0$ or $0 \rightarrow t \rightarrow t$. 
The heart $\mathscr{H}=\mathscr{A} \cap \Sigma \mathscr{B}$ is determined by

$$
\mathscr{H}=\operatorname{add}\left(x_{0} \oplus x_{1} \oplus x_{2}\right) .
$$

\section{Co-T-STructures}

The following definition was made in [5, def. 1.1.1] and [13, def. 2.4].

Definition 2.1 (Bondarko and Pauksztello). A co-t-structure in $\mathscr{T}$ is a pair ( $\mathscr{X}, \mathscr{Y}$ ) of full subcategories, closed under isomorphisms, direct sums, and direct summands, which satisfy the following conditions.

(i) $\Sigma^{-1} \mathscr{X} \subseteq \mathscr{X}$ and $\Sigma \mathscr{Y} \subseteq \mathscr{Y}$.

(ii) $\mathscr{T}(\mathscr{X}, \mathscr{Y})=0$.

(iii) For each object $t \in \mathscr{T}$ there is a triangle $x \rightarrow t \rightarrow y$ with $x \in \mathscr{X}, y \in \mathscr{Y}$.

The co-heart is $\mathscr{C}=\mathscr{X} \cap \Sigma^{-1} \mathscr{Y}$.

The co-t-structure is called bounded if

$$
\bigcup_{i \in \mathbb{Z}} \Sigma^{i} \mathscr{X}=\bigcup_{i \in \mathbb{Z}} \Sigma^{i} \mathscr{Y}=\mathscr{T}
$$

In contrast to t-structures, the objects $x$ and $y$ in Definition 2.1(iii) do not in general depend functorially on $t$, see [5, rmk. 1.2.2].

The following is the canonical example of a co-t-structure.

Example 2.2 (The standard co-t-structure). Let $R$ be a ring, $\mathscr{P}=\operatorname{Prj} R$ the category of projective left-modules over $R$. Let $\mathscr{X}$ and $\mathscr{Y}$ be the isomorphism closures in the bounded homotopy category $\mathscr{K}^{\mathrm{b}}(\mathscr{P})$ of the subsets

$$
\begin{aligned}
& \left\{\cdots \rightarrow 0 \longrightarrow 0 \longrightarrow 0 \rightarrow P^{0} \rightarrow P^{1} \rightarrow P^{2} \rightarrow \cdots \mid P^{i} \in \mathscr{P}, P^{i}=0 \text { for } i \gg 0\right\}, \\
& \left\{\cdots \rightarrow P^{-3} \rightarrow P^{-2} \rightarrow P^{-1} \rightarrow 0 \rightarrow 0 \rightarrow 0 \rightarrow \cdots \mid P^{i} \in \mathscr{P}, P^{i}=0 \text { for } i \ll 0\right\} .
\end{aligned}
$$

We will show that if $\mathscr{X}$ and $\mathscr{Y}$ are viewed as full subcategories, then $(\mathscr{X}, \mathscr{Y})$ is a bounded co-t-structure with co-heart $\mathscr{C}$ equivalent to $\mathscr{P}$.

Recall that $\mathscr{X}$ and $\mathscr{Y}$ are required to be closed under isomorphisms, direct sums, and direct summands. The two former properties are immediate, and the latter follows from the results in [14, secs. 3 and 4].

We next check the conditions in Definition 2.1. Conditions (i) and (ii) are clear. The triangle in Definition 2.1(iii) can be obtained by hard truncation of the object $t=\cdots \rightarrow P^{-2} \rightarrow$ $P^{-1} \rightarrow P^{0} \rightarrow P^{1} \rightarrow \cdots$ in $\mathscr{K}^{\mathrm{b}}(\mathscr{P})$, see Figure 2 in the introduction.

It is immediate that the co-t-structure $(\mathscr{X}, \mathscr{Y})$ is bounded.

Finally, it follows from [14, cor. 4.11] that the coheart $\mathscr{C}=\mathscr{X} \cap \Sigma^{-1} \mathscr{Y}$ is equivalent to $\mathscr{P}$.

The term silting set was coined in [9]. The following definition was made in [1, def. 2.1].

Definition 2.3. A silting subcategory $\mathscr{C}$ of $\mathscr{T}$ is a full subcategory, closed under isomorphisms, direct sums, and direct summands, which satisfies 
(i) $\mathscr{T}\left(\mathscr{C}, \Sigma^{>0} \mathscr{C}\right)=0$.

(ii) Each object in $\mathscr{T}$ can be obtained from $\mathscr{C}$ by taking finitely many (de)suspensions, triangles, and direct summands.

A silting object $s$ of $\mathscr{T}$ is an object such that add $(s)$ is a silting subcategory.

The following was proved in [12, cor. 5.9].

Theorem 2.4 (Mendoza Hernández et.al.). The map

$$
(\mathscr{X}, \mathscr{Y}) \mapsto \mathscr{C}=\mathscr{X} \cap \Sigma^{-1} \mathscr{Y}
$$

is a bijection between bounded co-t-structures and silting subcategories of $\mathscr{T}$.

Remark 2.5. The inverse map sends a silting subcategory $\mathscr{C}$ to a pair $(\mathscr{X}, \mathscr{Y})$ where $\mathscr{X}$ is the smallest full subcategory, closed under isomorphisms, direct sums, and direct summands, which is closed under $\Sigma^{-1}$ and contains $\mathscr{C}$. Similarly, $\mathscr{Y}$ is the smallest full subcategory, closed under isomorphisms, direct sums, and direct summands, which is closed under $\Sigma$ and contains $\Sigma \mathscr{C}$.

Example 2.6. We continue Example 1.4, so $\Lambda=\mathbb{C} A_{2}$ is the path algebra of the quiver $A_{2}$ from Equation (1.2) and $\mathscr{P}=\operatorname{prj} \Lambda$ is the category of finite dimensional projective left modules over $\Lambda$.

There is a bounded co-t-structure $(\mathscr{X}, \mathscr{Y})$ in $\mathscr{K}^{\mathrm{b}}(\mathscr{P})$ where $\mathscr{X}$ and $\mathscr{Y}$ are the isomorphism closures in $\mathscr{K}^{\mathrm{b}}(\mathscr{P})$ of the subsets in Equation (2.1); this is shown by the same method as in Example 2.2.

Recall that $\Lambda$ also has a bounded derived category $\mathscr{D}^{\mathrm{b}}(\mathscr{M})$, see Example 1.4. Since $\Lambda$ has global dimension 1 , the triangulated categories $\mathscr{K}^{\mathrm{b}}(\mathscr{P})$ and $\mathscr{D}^{\mathrm{b}}(\mathscr{M})$ are equivalent, so $\mathscr{K}^{\mathrm{b}}(\mathscr{P})$ has the Auslander-Reiten quiver shown in Equation (1.3). We redraw the quiver, this time with red and green vertices showing $\mathscr{X}$ and $\mathscr{Y}$.

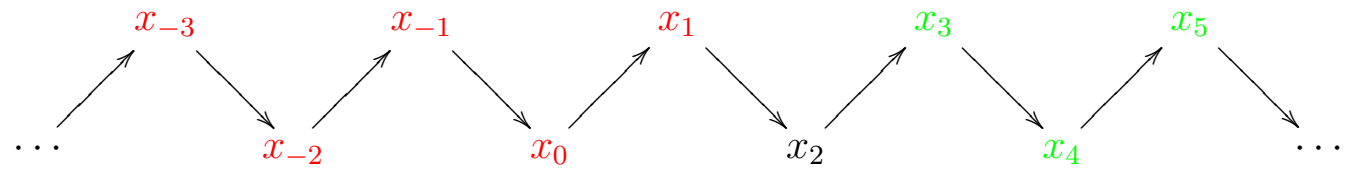

Note that $x_{2}$ is neither in $\mathscr{X}$ nor $\mathscr{Y}$. Indeed, if we abuse notation to confuse isomorphism classes with individual objects, then we can set $t=x_{2}$ and the triangle in Definition 2.1(iii) becomes $x_{1} \rightarrow x_{2} \rightarrow x_{3}$.

The coheart $\mathscr{C}=\mathscr{X} \cap \Sigma^{-1} \mathscr{Y}$ is determined by

$$
\mathscr{C}=\operatorname{add}\left(x_{0} \oplus x_{1}\right) .
$$

Theorem 2.4 implies that $\mathscr{C}$ is a silting subcategory of $\mathscr{K}^{\mathrm{b}}(\mathscr{P})$. The corresponding isomorphism class of silting objects is $x_{0} \oplus x_{1}$.

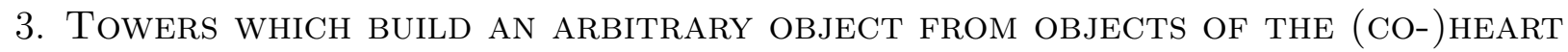

The following two results were proved in [4, p. 34] and [5, prop. 1.5.6]. A wavy arrow $s \sim t$ denotes a morphism $s \rightarrow \Sigma t$. 
Proposition 3.1 (Beilinson, Bernstein, and Deligne). Let $(\mathscr{A}, \mathscr{B})$ be a bounded t-structure in $\mathscr{T}$ with heart $\mathscr{H}$. For each object $t \in \mathscr{T}$, there is an integer $n \geqslant 1$ and a diagram consisting of triangles,

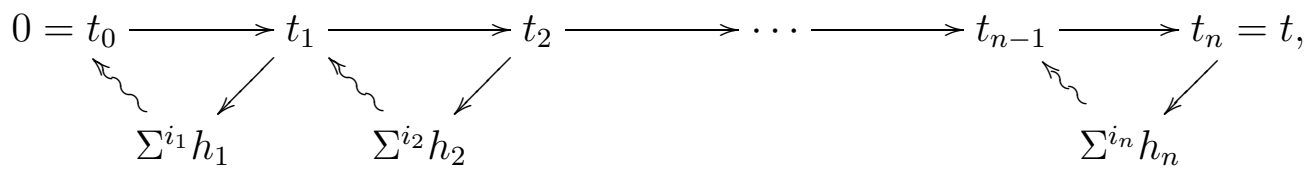

with $h_{m} \in \mathscr{H}$ for each $m$ and $i_{1}>i_{2}>\cdots>i_{n}$.

Proposition 3.2 (Bondarko). Let ( $\mathscr{X}, \mathscr{Y})$ be a bounded co-t-structure in $\mathscr{T}$ with co-heart $\mathscr{C}$. For each object $t \in \mathscr{T}$, there is an integer $n \geqslant 1$ and a diagram consisting of triangles,

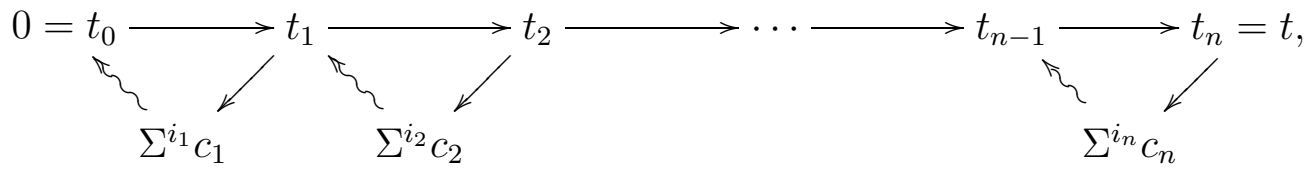

with $c_{m} \in \mathscr{C}$ for each $m$ and $i_{1}<i_{2}<\cdots<i_{n}$.

Example 3.3. Consider the t-structure in Example 1.2. If $t \in \mathscr{D}^{\mathrm{b}}(\mathscr{M})$ is given, then there is a diagram as in Proposition 3.1 where the objects $\sum^{i_{m}} h_{m}$ are of the form $\Sigma^{i} \mathrm{H}^{-i}(t)$. The diagram expresses that $t$ can be built from its cohomology modules $\mathrm{H}^{-i}(t)$.

Example 3.4. Consider the co-t-structure in Example 2.2. If $t=\cdots \rightarrow P^{-2} \rightarrow P^{-1} \rightarrow$ $P^{0} \rightarrow P^{1} \rightarrow \cdots$ in $\mathscr{K}^{\mathrm{b}}(\mathscr{P})$ is given, then there is a diagram as in Proposition 3.2 where the objects $\Sigma^{i_{m}} c_{m}$ are of the form $\Sigma^{i} P^{-i}$. The diagram expresses that $t$ can be built from its constituent modules $P^{-i}$.

\section{Categories Skewed towards T- OR CO-T-Structures}

In this section, $d$ is a fixed integer.

Definition 4.1. If $\mathscr{T}$ is $\mathbb{C}$-linear, then an object $s \in \mathscr{T}$ is called $d$-spherical if there is an isomorphism

$$
\mathscr{T}\left(s, \Sigma^{*} s\right) \cong \mathbb{C}[X] /\left(X^{2}\right)
$$

of graded algebras where $\operatorname{deg} X=d$.

Remark 4.2. If $d \neq 0$, then $s$ is $d$-spherical if and only if there are isomorphisms of $\mathbb{C}$-vector spaces

$$
\mathscr{T}\left(s, \Sigma^{i} s\right) \cong \begin{cases}\mathbb{C} & \text { for } i \in\{0, d\} \\ 0 & \text { otherwise. }\end{cases}
$$

The following was proved in [10, thm. 2.1].

Theorem 4.3 (Keller, Yang, and Zhou). There is a triangulated category $\mathscr{S}_{d}$ which is algebraic, $\mathbb{C}$-linear with finite dimensional Hom-spaces, and contains a $d$-spherical object $s$ such that each object in $\mathscr{S}_{d}$ can be obtained from s by taking finitely many (de)suspensions, triangles, and direct summands.

Up to triangulated equivalence, $\mathscr{S}_{d}$ is unique.

The following was proved in [7, thm. A]. It clearly implies that if $d \leqslant 0$, then bounded co-t-structures are more useful than bounded t-structures for the study of $\mathscr{S}_{d}$. 
Theorem 4.4 (Holm, J, and Yang). If $d \leqslant 0$ then $\mathscr{S}_{d}$ has no bounded $t$-structures. It has one familiy of bounded co-t-structures, all of which are (de)suspensions of a canonical one.

If $d \geqslant 1$ then $\mathscr{S}_{d}$ has no bounded co-t-structures. It has one familiy of bounded $t$-structures, all of which are (de)suspensions of a canonical one.

\section{The BIJECTIONS OF KÖNIG AND YANG}

Definition 5.1. A simple minded collection in $\mathscr{T}$ is a set $\left\{t_{1}, \ldots, t_{n}\right\}$ of objects of $\mathscr{T}$ with the following properties.

(i) $\mathscr{T}\left(t_{i}, \Sigma^{<0} t_{j}\right)=0$ for all $i$ and $j$.

(ii) $\mathscr{T}\left(t_{i}, t_{i}\right)$ is a division ring for each $i$ and $\mathscr{T}\left(t_{i}, t_{j}\right)=0$ when $i \neq j$.

(iii) Each object in $\mathscr{T}$ can be obtained from $t_{1}, \ldots, t_{n}$ by taking finitely many (de)suspensions, triangles, and direct summands.

Let $\Lambda$ be a finite dimensional $\mathbb{C}$-algebra, $\mathscr{M}=\bmod \Lambda$ the category of finite dimensional left modules over $\Lambda$, and $\mathscr{P}=\operatorname{prj} \Lambda$ the category of finite dimensional projective left modules over $\Lambda$. The following was proved in [11, thm. (6.1)]. We interpret it as indicating that the role of t-structures in derived categories is taken over by co-t-structures in homotopy categories.

Theorem 5.2. There are bijections between the following sets.

(i) Bounded t-structures in $\mathscr{D}^{\mathrm{b}}(\mathscr{M})$ whose hearts are length categories ("length category" means that each object has finite length).

(ii) Bounded co-t-structures in $\mathscr{K}^{\mathrm{b}}(\mathscr{P})$.

(iii) Isomorphism classes of simple minded collections in $\mathscr{D}^{\mathrm{b}}(\mathscr{M})$.

(iv) Isomorphism classes of basic silting objects in $\mathscr{K}^{\mathrm{b}}(\mathscr{P})$ (basic means no repeated indecomposable summands).

Remark 5.3. There is an extensive body of work on the bijections of Theorem 5.2 which predates [11, see [1], [2], [3], [5], [6], [8], [9], and [12]. The contributions of these papers to Theorem 5.2 are explained in the introduction to [11].

Remark 5.4. The proof of Theorem 5.2 occupies a significant part of [11], and we only show how some of the bijections are defined.

(i) to (iii): Let $(\mathscr{A}, \mathscr{B})$ be a bounded t-structure in $\mathscr{D}^{\mathrm{b}}(\mathscr{M})$ whose heart $\mathscr{H}=\mathscr{A} \cap \sum \mathscr{B}$ is a length category. Take a simple object from each isomorphism class of simple objects in $\mathscr{H}$. This gives a simple minded system in $\mathscr{D}^{\mathrm{b}}(\mathscr{M})$, see [11, sec. 5.3].

(ii) to (iv): Let $(\mathscr{X}, \mathscr{Y})$ be a bounded co-t-structure in $\mathscr{K}^{\mathrm{b}}(\mathscr{P})$. The co-heart $\mathscr{C}=\mathscr{X} \cap$ $\Sigma^{-1} \mathscr{Y}$ is a silting subcategory by Theorem 2.4, and there is a silting object $s$ such that $\mathscr{C}=\operatorname{add}(s)$, see [11, sec. 5.2].

(i) to (ii): Let $(\mathscr{X}, \mathscr{Y})$ be a bounded co-t-structure in $\mathscr{K}^{\mathrm{b}}(\mathscr{P})$. Set

$$
\begin{gathered}
\mathscr{A}=\left\{a \in \mathscr{D}^{\mathrm{b}}(\mathscr{M}) \mid \operatorname{Hom}_{\mathscr{D}^{\mathrm{b}}(\mathscr{M})}(x, a)=0 \text { for each } x \in \mathscr{X}\right\}, \\
\mathscr{B}=\left\{b \in \mathscr{D}^{\mathrm{b}}(\mathscr{M}) \mid \operatorname{Hom}_{\mathscr{D}^{\mathrm{b}}(\mathscr{M})}(y, b)=0 \text { for each } y \in \mathscr{Y}\right\}
\end{gathered}
$$


and view these two sets as full subcategories of $\mathscr{D}^{\mathrm{b}}(\mathscr{M})$. Then $(\mathscr{A}, \mathscr{B})$ is a bounded tstructure in $\mathscr{D}^{\mathrm{b}}(\mathscr{M})$ with length heart, see [11, sec. 5.7].

\section{The Silting mutation of Aihara And Iyama}

Silting mutation is an operation which changes one silting subcategory into another. By virtue of Theorem 2.4, it can be viewed as changing one bounded co-t-structure into another. This leads to the definition of the so-called silting quiver of the triangulated category $\mathscr{T}$ which shows how silting subcategories, and hence co-t-structures, fit together inside $\mathscr{T}$.

In this section, $\mathscr{T}$ is $\mathbb{C}$-linear with finite dimensional Hom-spaces and split idempotents, and $m=m_{0} \oplus m_{1}$ is a basic silting object of $\mathscr{T}$ with $m_{0}$ indecomposable. The following definition and theorem are special cases of [1, sec. 2.4].

Definition 6.1 (Aihara and Iyama). Let $r \stackrel{\rho}{\rightarrow} m_{0} \stackrel{\lambda}{\rightarrow} \ell$ be a minimal right add $\left(m_{1}\right)$ approximation and a minimal left $\operatorname{add}\left(m_{1}\right)$-approximation of $m_{0}$. Complete these morphisms to triangles

in $\mathscr{T}$ and set

$$
m_{0}^{\sim} \rightarrow r \stackrel{\rho}{\rightarrow} m_{0} \quad, \quad m_{0} \stackrel{\lambda}{\rightarrow} \ell \rightarrow m_{0}^{\dagger}
$$

$$
\mu^{-}\left(m, m_{1}\right)=m_{0}^{\sim} \oplus m_{1}, \mu^{+}\left(m, m_{1}\right)=m_{0}^{\dagger} \oplus m_{1} .
$$

These are called right and left silting mutations of $m$.

Theorem 6.2 (Aihara and Iyama). $\quad$ (i) The silting mutations $\mu^{-}\left(m, m_{1}\right)$ and $\mu^{+}\left(m, m_{1}\right)$ are basic silting objects of $\mathscr{T}$.

(ii) Right and left silting mutations are inverse in the sense that

$$
\mu^{-}\left(\mu^{+}\left(m, m_{1}\right), m_{1}\right) \cong m, \mu^{+}\left(\mu^{-}\left(m, m_{1}\right), m_{1}\right) \cong m .
$$

The following is a special case of [1, def. 2.41].

Definition 6.3 (Aihara and Iyama). The silting quiver of $\mathscr{T}$ has a vertex for each isomorphism class of basic silting objects of $\mathscr{T}$, and an arrow $[m] \rightarrow\left[m^{*}\right]$ if $m^{*}$ is a left silting mutation of $m$, where square brackets denote isomorphism class.

Remark 6.4. The silting quiver gives a picture of how silting mutation moves from one silting object to another, hence from one silting subcategory to another. By virtue of Theorem [2.4, it gives a picture of how silting mutation moves from one bounded co-t-structure to another.

Example 6.5. We continue Example 2.6, so $\Lambda=\mathbb{C} A_{2}$ is the path algebra of the quiver $A_{2}$ from Equation (1.2). The bounded homotopy category $\mathscr{K}^{\mathrm{b}}(\mathscr{P})$ has the Auslander-Reiten quiver shown in Equation (2.2).

Recall from Example 2.6 that $\mathscr{K}^{\mathrm{b}}(\mathscr{P})$ has the isomorphism class of silting objects $x_{0} \oplus x_{1}$. Indeed, $x_{0} \oplus x_{1}$ is a vertex in the silting quiver of $\mathscr{K}^{\mathrm{b}}(\mathscr{P})$. The full quiver was determined in [1, exa. 2.45], see Figure 3. As the quiver shows, there is a left silting mutation of $x_{0} \oplus x_{1}$ which gives $x_{0} \oplus x_{4}$. The isomorphism classes of silting objects $x_{0} \oplus x_{1}$ and $x_{0} \oplus x_{4}$ give rise to silting subcategories $\operatorname{add}\left(x_{0} \oplus x_{1}\right)$ and $\operatorname{add}\left(x_{0} \oplus x_{4}\right)$ which, under the bijection of Theorem 2.4, correspond to two bounded co-t-structures. The first of these is shown on the AR quiver 


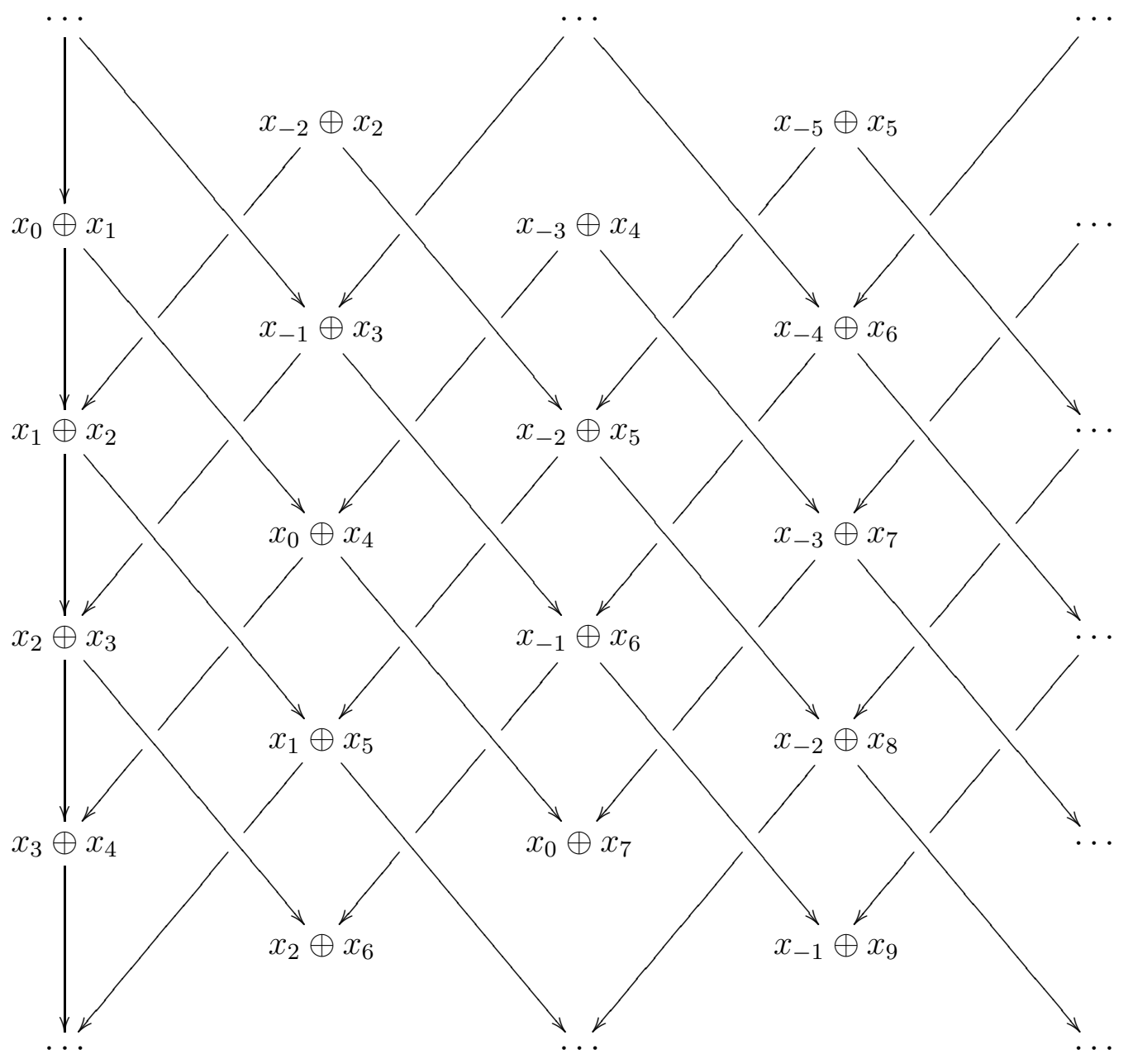

FiguRE 3 . The silting quiver of the bounded homotopy category $\mathscr{K}^{\mathrm{b}}(\mathscr{P})$ where $\mathscr{P}$ is the category of finite dimensional projective modules over $\mathbb{C} A_{2}$.

of $\mathscr{K}^{\mathrm{b}}(\mathscr{P})$ in Equation (2.2) . The second co-t-structure $\left(\mathscr{X}^{\prime}, \mathscr{Y}^{\prime}\right)$ can be shown as follows, where the red and green vertices show $\mathscr{X}^{\prime}$ and $\mathscr{Y}^{\prime}$.

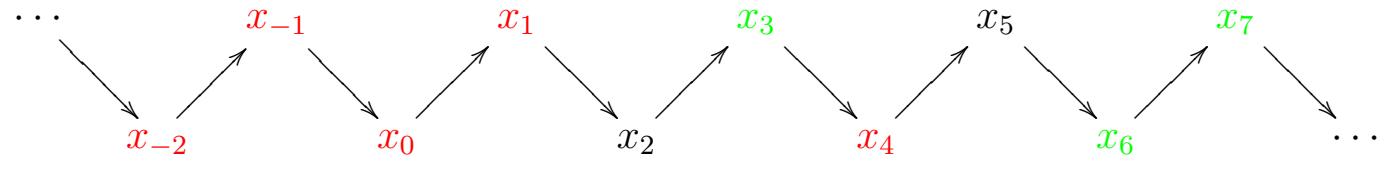

Acknowledgement. I thank Kiyoshi Igusa, Alex Martsinkovsky, and Gordana Todorov for inviting me to speak at the Maurice Auslander International Conference and to write this survey.

\section{REFERENCES}

[1] T. Aihara and O. Iyama, Silting mutation in triangulated categories, J. London Math. Soc. (2) 85 (2012), 633-668.

[2] S. Al-Nofayee, Simple objects in the heart of a t-structure, J. Pure Appl. Algebra 213 (2009), 54-59. 
[3] I. Assem, M. J. Souto Salorio, and S. Trepode, Ext-projectives in suspended subcategories, J. Pure Appl. Algebra 212 (2008), 423-434.

[4] A. A. Beilinson, J. Bernstein, and P. Deligne, Faisceaux pervers, Astérisque 100 (1982) (Vol. 1 of the proceedings of the conference "Analysis and topology on singular spaces", Luminy, 1981).

[5] M. V. Bondarko, Weight structures vs. t-structures; weight filtrations, spectral sequences, and complexes (for motives and in general), J. K-Theory 6 (2010), 387-504.

[6] A. B. Buan, I. Reiten, and H. Thomas, From m-clusters to m-noncrossing partitions via exceptional sequences, Math. Z. 271 (2012), 1117-1139.

[7] T. Holm, P. Jørgensen, and D. Yang, Sparseness of t-structures and negative Calabi-Yau dimension in triangulated categories generated by a spherical object, Bull. London Math. Soc. 45 (2013), 120-130.

[8] B. Keller and P. Nicolas, Weight structures and simple $d g$ modules for positive dg algebras, Internat. Math. Res. Notices 2013, no. 5, 1028-1078.

[9] B. Keller and D. Vossieck, Aisles in derived categories, Bull. Soc. Math. Belg. Sér. A 40 (1988), 239-253.

[10] B. Keller, D. Yang, and G. Zhou, The Hall algebra of a spherical object, J. London Math. Soc. (2) 80 (2009), 771-784.

[11] S. König and D. Yang, Silting objects, simple-minded collections, t-structures and co-t-structures for finite-dimensional algebras, Doc. Math. 19 (2014), 403-438.

[12] O. Mendoza Hernández, E. C. Sáenz Valadez, V. Santiago Vargas, M. J. Souto Salorio, AuslanderBuchweitz context and co-t-structures, Appl. Categ. Structures 21 (2013), 417-440.

[13] D. Pauksztello, Compact corigid objects in triangulated categories and co-t-structures, Cent. Eur. J. Math. 6 (2008), 25-42.

[14] O. M. Schnürer, Homotopy categories and idempotent completeness, weight structures and weight complex functors, preprint (2011). math.CT/1107.1227v1.

School of Mathematics and Statistics, Newcastle University, Newcastle upon Tyne Ne1 7RU, United Kingdom

E-mail address: peter.jorgensen@ncl.ac.uk

$U R L:$ http://www.staff.ncl.ac.uk/peter.jorgensen 